'Departamento de Medicina Intensiva, Escuela de Medicina, Facultad de Medicina, Pontificia Universidad Católica de Chile. Santiago, Chile.

${ }^{2}$ Unidad de Pacientes Críticos Complejo Asistencial Doctor Sótero del Río. Santiago, Chile.

Conflictos de intereses: ninguno por declarar.

Recibido el 25 de octubre de 2013, aceptado el 22 de enero de 2014.

Correspondencia a: Dra. Carolina Ruiz Hospital Clínico Pontificia Universidad Católica de Chile, Departamento de Medicina Intensiva.

Marcoleta 367, Santiago, Chile. Teléfono: 5623543746 cruizbalart@gmail.com

\section{Reanimación con fluidos y hemoderivados en trauma}

\author{
CAROLINA RUIZ ${ }^{1,2}$, MAX ANDRESEN ${ }^{1}$
}

\section{Resuscitation with fluids and blood products in trauma}

The leading cause of preventable death in trauma is uncontrolled bleeding. Some of these deaths may be secondary to the Acute Coagulopathy of Trauma, which is present in $30 \%$ of patients on admission to hospital. In recent years, Damage Control Resuscitation has been developed, which aims to give a hemostatic resuscitation and avoid deaths caused by bleeding. This strategy considers control of bleeding with damage control surgery or interventional procedures, early transfusion of all blood products, permissive hypotension and limited use of crystalloids. Several studies have reported that early transfusion of all blood products, with a ratio close to 1:1, may be associated with lower mortality and less requirements of total transfusions in trauma patients at risk of massive bleeding and massive transfusion. These findings must be corroborated in randomized controlled trials, along with determining the optimum ratio between blood products. In stable patients, a restrictive transfusion strategy is safe and appropriate.

(Rev Med Chile 2014; 142: 758-766)

Key words: Blood coagulation disorders; Multiple Trauma; Resuscitation.
$\mathrm{E}$ 1 trauma es la principal causa de muerte en menores de 40 años, generando $10 \%$ de la mortalidad mundial. Hasta $40 \%$ de estas muertes son secundarias a sangrado no controlado, constituyendo la causa más frecuente de muerte intrahospitalaria y, por lo tanto, una causa prevenible ${ }^{1}$.

Durante los últimos años la reanimación del trauma ha cambiado en forma importante, especialmente en el aporte de fluidos y hemoderivados. Tradicionalmente estos pacientes recibían gran cantidad de cristaloides y sólo eran transfundidos después de varias horas de evolución. La reanimación propuesta actualmente para el trauma grave corresponde a la "reanimación con control de daños", cuyo objetivo es realizar una resucitación hemostática y así evitar la muerte por sangrado. La reanimación con control de daños considera el control del sangrado, el aporte precoz de todos los hemoderivados, la hipotensión permisiva y el aporte controlado de cristaloides ${ }^{2}$.
El presente artículo revisa el aporte de hemoderivados y fluidos en trauma, en el contexto de la reanimación con control de daños.

\section{Reanimación con control de daño}

Hasta hace algunos años los pacientes con trauma grave eran reanimados con gran cantidad de cristaloides isotónicos y transfusión de glóbulos rojos (GR), con escaso aporte de plasma, lo que generaba hemodilución y mayor coagulopatía, pudiendo presentarse posteriormente hipertensión intraabdominal, síndrome compartimental abdominal y deterioro respiratorio ${ }^{3}$. Durante los últimos años se ha desarrollado la reanimación con control de daños (RCD), cuyos objetivos son minimizar el sangrado, restablecer la perfusión, prevenir la coagulopatía y evitar las disfunciones orgánicas. De esta forma, se busca disminuir la mortalidad del trauma, especialmente la muerte por sangrado no controlado ${ }^{2}$. El eje central de la 
RCD es el control precoz del sangrado mediante procedimientos quirúrgicos (cirugía de control de daños) e intervencionales. Esta estrategia, además, considera la restauración de la volemia y del transporte de oxígeno, junto con la corrección de la coagulopatía. Esto se consigue mediante el aporte precoz de todos los hemoderivados, la hipotensión permisiva, el aporte controlado de cristaloides y la corrección de la hipotermia y la acidosis (Figura 1).

El origen de la RCD es la cirugía de control de daños. Ésta plantea que el manejo quirúrgico inicial del trauma debe enfocarse en el control del sangrado y de la contaminación (lesiones de víscera hueca), realizando las reconstrucciones definitivas una vez que el paciente se ha estabilizado y ha recuperado su homeostasis ${ }^{4}$. Su objetivo es restaurar la fisiología del paciente difiriendo la restauración de la anatomía, evitando las complicaciones de cirugías prolongadas, especialmente el desarrollo de la triada maligna del trauma (hipotermia, acidosis y coagulopatía). Actualmente, se considera que la cirugía de control de daños es un estándar de manejo del trauma grave, reportándose una mejoría en la sobrevida con esta estrategia ${ }^{5}$.

\section{Coagulopatía del trauma}

Uno de los objetivos de la RCD es prevenir o corregir el desarrollo de coagulopatía. La coagulopatía es un marcador de gravedad en trauma, aso- ciándose a mayor sangrado, más complicaciones e incluso la muerte por exanguinación. Tradicionalmente, se consideraba que la coagulopatía del trauma era secundaria a hemodilución de factores de coagulación, acidosis e hipotermia, desarrollándose varias horas después del ingreso al hospital ${ }^{6}$. En los últimos años se ha reconocido que $25 \%$ a $30 \%$ de los pacientes de trauma presentan alteraciones de las pruebas de coagulación al ingreso al hospital, antes de haber recibido gran cantidad de fluidos y antes de desarrollar hipotermia y acidosis ${ }^{7,8}$. Ante estos hallazgos, se ha planteado que precozmente existiría una coagulopatía propia del trauma, que se ha denominado "ACoT", del inglés acute coagulopathy of trauma. Esta coagulopatía aguda del trauma (CAT) es un marcador de mal pronóstico, asociándose a mayor mortalidad frente a lesiones de igual severidad. Asimismo, la CAT se asocia a más requerimientos transfusionales, más días de UCI y ventilación mecánica, y también a mayor desarrollo de falla orgánica múltiple ${ }^{9}$.

La fisiopatología exacta de la CAT aún no está clara, pero probablemente sea multifactorial y esté relacionada con la severidad del trauma, ya que a mayor Injury Severity Score (ISS) hay mayor incidencia de CAT. Se han postulado 2 mecanismos para explicar su desarrollo. El primero se relaciona con la hipoperfusión que pueden presentar los pacientes con sangrado masivo, lo que activaría al anticoagulante natural proteína C (PCA). La PCA inactiva a los factores VIII y $\mathrm{V}$, generando además un aumento de la fibrinólisis ${ }^{10,11}$. Este aumento de
OBJETIVOS

$\rightarrow$ Detener el sangrado a la brevedad

$\rightarrow$ Resuscitación adecuada $\rightarrow$ resuscitación hemostática

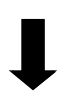

DO2 adecuado (volemia + transportador de O2)

Evitar/corregir la coagulopatía del trauma
Corrección de la coagulopatía

Perfusión adecuada

\section{COMPONENTES}

- Aporte de fluidos adecuado (resucitación hipotensiva)

- Aporte de transfusiones adecuado (corrección coagulopatía)

- Corrección hipotermia

- Corrección acidosis

- Procedimientos intervencionales

- Círugia control de daños (hemostasia + control contaminación)

Figura 1. Objetivos y componentes de la reanimación con control de daños. DO2 (transporte de oxígeno). La reanimación con control de daños implica una resucitación hemostática, en que exista un equilibrio entre el control del sangrado y una perfusión adecuada. 
la fibrinólisis sería potenciado por un incremento del activador del plasminógeno tisular (TPA), secundario a daño tisular. El segundo mecanismo plantea que por daño endotelial secundario al trauma se expondría factor tisular, que finalmente resultaría en el desarrollo de coagulación intravascular diseminada $(\mathrm{CID})^{12}$. Este mecanismo ha sido refutado por varios autores, que consideran que la hipoperfusión es el punto clave en el desarrollo de la CAT, denominándola incluso como AcoTS (Acute coagulopathy of Trauma-Shock) ${ }^{13}$. Además, se ha reportado la ausencia de trombosis microvascular, aspecto característico de $\mathrm{CID}^{14}$. Adicionalmente, se ha planteado que, dada la interacción que existe entre activación de la coagulación e inflamación, la CAT podría ser parte del estado pro inflamatorio de las etapas iniciales del trauma severo. Independiente de los mecanismos que generan la CAT, esta puede ser potenciada por hemodilución, acidosis e hipotermia, si los pacientes no reciben un manejo adecuado ${ }^{10}$ (Figura 2).

La CAT debe considerarse en todo paciente de trauma, especialmente si el mecanismo fue de alta energía, existan evidencias de sangrado importante (compromiso hemodinámico e hipoperfusión) y presencia de lesiones severas ${ }^{11}$. Aún no hay criterios diagnósticos claros para la CAT, lo que dificulta su reconocimiento y tratamiento precoz. La mayoría de los autores han utilizando la prolongación del tiempo de protrombina (TP) y del tiempo de tromboplastina activada (TTPa $)^{6}$. Si bien estos son exámenes sencillos y ampliamente disponibles, son test que miden la hemostasia del plasma y no de la sangre, evaluando sólo los primeros 60 segundos de la formación del coágulo (proceso que dura entre 15 a $30 \mathrm{~min}$ ). Además, se realizan a $37^{\circ} \mathrm{C}$ y en $\mathrm{pH} 7,5$, sin considerar la presencia de hipotermia, acidosis e hipocalcemia ${ }^{12}$. Se ha planteado el uso de tromboelastografía (TEG) y tromboelastometría (ROTEM) para el diagnóstico de CAT. Estos métodos determinan las propiedades fibro elásticas del coágulo, evaluando el proceso de coagulación y fibrinólisis en forma completa ${ }^{3}$. Se ha reportado que ambos permitirían evaluar la hiperfibrinolisis asociada a trauma y que podrían ser útiles en predecir los requerimientos transfusionales ${ }^{15,16}$. Sin embargo, su uso actualmente presenta limitaciones, como la disponibilidad de equipos, la necesidad de capacitación del personal, el tiempo necesario para obtener resultados (30 min) y la falta de puntos de corte para el diagnóstico de CAT.

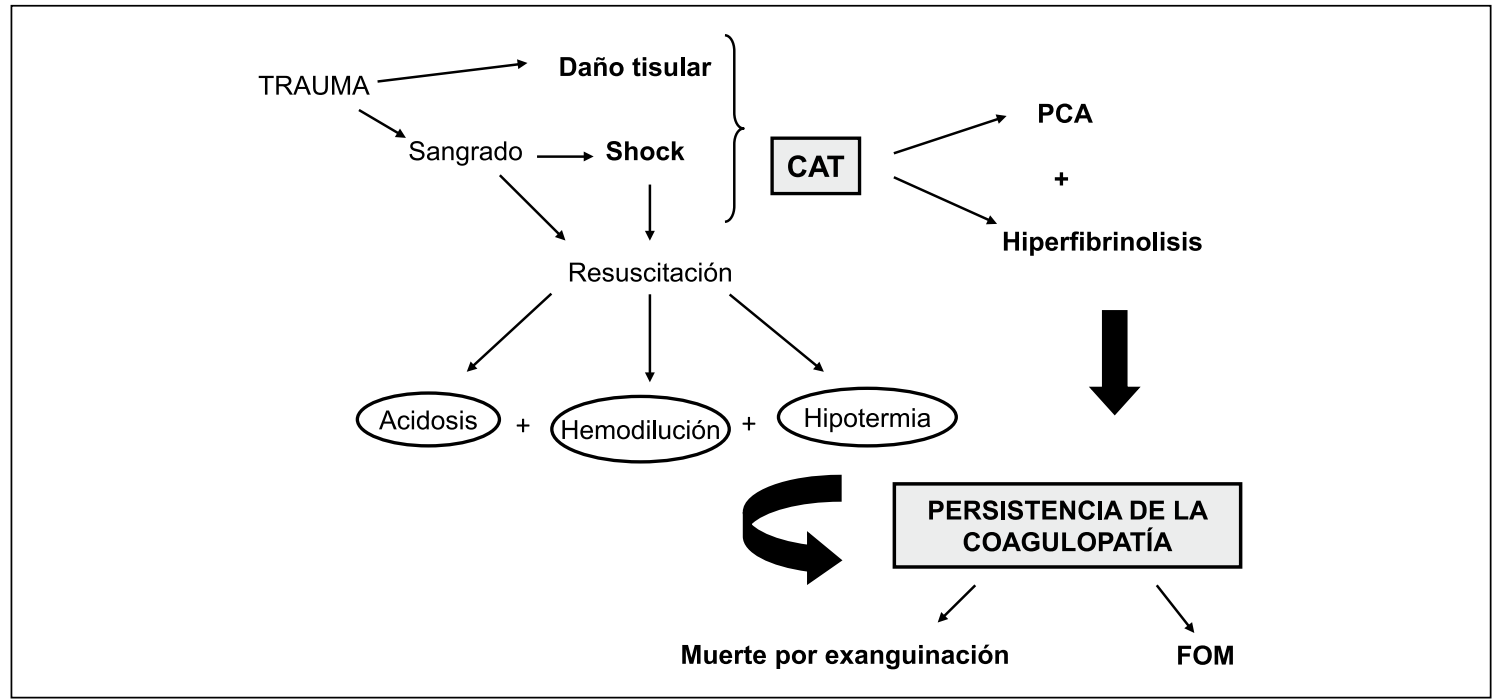

Figura 2. Fisiopatología de la coagulopatía aguda del trauma. CAT (coagulopatía aguda del trauma). PCA (Proteína C activada). FOM (falla orgánica múltiple). Se ha postulado que para el desarrollo de CAT es necesario que exista hipoperfusión (secundaria a sangrado) además de trauma. Esta hipoperfusión activaría a la proteína C (APC), que es un anticoagulante natural que además induce fibrinolisis. Por otra parte, en el trauma hay un aumento de la fibrinolisis secundaria a exposición del factor tisular. Además de este aumento de los anticogulantes naturales y de la fibrinolisis, la coagulación podría alterarse aún más debido a una reanimación inadecuada, que resulte en hemodilución, hipotermia y acidosis. Si estas alteraciones no se corrigen, el paciente puede fallecer producto de un sangrado no controlado o presentar complicaciones como el desarrollo de FOM. 


\section{Hipotensión permisiva}

La hipotensión permisiva o resucitación hipotensora consiste en limitar la presión arterial (PA) restringiendo el aporte de fluidos, principalmente de cristaloides, mientras no se controle el sangrado $^{17}$. Un aporte generoso de fluidos durante la reanimación puede inducir mayor sangrado y más coagulopatía debido a hemodilución. Además, el aumento de la PA producto del aporte de fluidos podría generar el desprendimiento de un trombo en formación, con el consiguiente mayor sangrado ${ }^{3}$.

Existe evidencia experimental ${ }^{18,19}$ y clínica que muestra resultados positivos con esta estrategia. Bickell $^{20}$, en un estudio prospectivo y controlado, evaluó el efecto de la reanimación hipotensora en pacientes con trauma penetrante de torso y compromiso hemodinámico, desde el pre hospitalario hasta el ingreso a pabellón. El grupo intervenido requirió menos cristaloides, tuvo una mejor sobrevida y una menor estadía hospitalaria. Cabe destacar, que no hubo diferencia en la PA entre el grupo intervenido y el control, tanto en el pre hospitalario, urgencia o al inicio de la cirugía. Estos resultados favorables no han sido corroborados por otros autores en estudios similares, pero con inclusión de pacientes con trauma contuso ${ }^{21}$. Recientemente se publicaron los resultados preliminares de un estudio que evaluó la estrategia de resucitación hipotensora en pabellón (cirugía de urgencia en trauma), encontrando mejoría en la mortalidad en el postoperatorio inmediato pero no a 30 días $^{22}$.

Aún no existe claridad sobre qué metas de presión recomendar en el contexto de la resucitación hipotensora. Algunos autores recomiendan metas tan bajas como una PA sistólica de 60 a $70 \mathrm{~mm} \mathrm{Hg}$ en trauma penetrante ${ }^{23}$. Tampoco está claro si esta estrategia es aplicable al trauma contuso. Lo que sí está claro, es que el aporte de fluidos puede ser deletéreo durante la reanimación inicial del trauma. Estudios en urgencia ${ }^{24}$ y en el pre hospitalario ${ }^{25}$ han reportado que el aporte de cristaloides es un factor de riesgo independiente de la mortalidad.

El efecto positivo de la resucitación hipotensora probablemente está dado por limitar el desarrollo de coagulopatía al evitar la hemodilución. En este sentido, el elemento más importante de esta estrategia es limitar el aporte de fluidos, con lo que secundariamente se limita la PA. Sin embargo, debe existir un equilibrio entre limitar la PA y el aporte de fluidos, y el desarrollo de hipoperfusión. Además, esta estrategia sólo debe mantenerse mientras se consigue controlar el sangrado, lo que debe ocurrir en el menor tiempo posible. En el estudio de Bickell ${ }^{20}$, los pacientes eran llevados a pabellón en un lapso entre 75 y 90 min desde el accidente.

\section{Aporte de fluidos en trauma}

Los cristaloides isotónicos han sido por décadas el estándar de manejo en la reanimación inicial del trauma, aunque actualmente existe evidencia de que su uso podría ser deletéreo, especialmente si el sangrado no ha sido controlado. Además, su uso podría agravar el estado pro inflamatorio inicial del trauma, así como generar acidosis hiperclorémica $^{26}$.

El aporte de suero salino hipertónico (SSH) ha sido estudiado extensamente en trauma. Se ha postulado que su uso podría tener efectos antiinflamatorios inmunomoduladores, lo que podría asociarse a menos disfunciones orgánicas y menor mortalidad. Además, su administración implica una reanimación con menor volumen, lo que también podría ser positivo al evitar las complicaciones asociadas al aporte de grandes volúmenes $^{27}$. El SSH arrastra agua desde el intersticio al intravascular, por lo que su aporte se asocia a un aumento en la volemia de 9 a 10 veces el volumen infundido. Estudios experimentales ${ }^{28}$ y estudios fisiológicos ${ }^{29,30}$ han reportado resultados positivos, especialmente la disminución de marcadores pro inflamatorios. Sin embargo, 2 estudios clínicos aleatorizados no demostraron mejoría en morbilidad o mortalidad con el uso de SSH 7,5\% (250 $\mathrm{ml}$ en $15 \mathrm{~min}$ ) versus cristaloides en el pre hospitalario ${ }^{31,32}$. Ambos estudios fueron suspendidos precozmente por futilidad.

Los coloides, especialmente los almidones (HES), también han sido evaluados en la reanimación del trauma. Dado su mayor poder oncótico, se requiere aportar menos volumen ${ }^{26}$. A pesar de esto, no se ha encontrado que su uso se asocie a resultados clínicos positivos en trauma ni en otros pacientes críticos, en comparación con cristaloides. Perne ${ }^{33}$ demostró que el uso de HES se asociaba a más mortalidad y mayores requerimientos de terapia de reemplazo renal en pacientes con sepsis 
severa. Myburgh ${ }^{34}$ encontró que el uso de HES se asoció a mayor necesidad de terapia de reemplazo renal en una población mixta de pacientes críticos. Dados estos resultados, actualmente se plantea limitar el uso de estos fluidos en pacientes críticos, incluido el trauma ${ }^{35,36}$.

Finalmente, el aporte de albúmina a $4 \%$ no demostró beneficios en mortalidad o morbilidad en un gran estudio clínico aleatorizado en una población mixta de pacientes críticos ${ }^{37}$. En este mismo estudio, se encontró que el uso de albúmina se asociaba a una tendencia a mayor mortalidad en el subgrupo de trauma.

Por el momento, parece que los fluidos más seguros y costo efectivos en trauma son los cristaloides isotónicos. Sin embargo, como ya se ha señalado, su uso no está exento de complicaciones, por lo que deben administrarse en forma controlada, especialmente durante la reanimación inicial.

\section{Aporte de transfusiones en pacientes con trauma}

Recientemente se han publicado varios estudios $^{38-47}$ que han reportado que un aporte precoz y generoso de todos los hemoderivados, con una relación entre ellos cercana a 1:1, puede asociarse a menor mortalidad y disminución de las transfusiones totales en pacientes con trauma con riesgo de transfusión masiva (TM.) La TM traduce sangrado masivo, es decir, la pérdida de más de una volemia (70 ml/kg de peso) en $24 \mathrm{~h}$. El sangrado masivo implica mayor morbilidad y mortalidad $^{10}$. La TM se define clásicamente como la transfusión de 10 o más unidades de GR en $24 \mathrm{~h}$. Este concepto ha sido criticado por varios autores, planteando que es una definición arbitraria ${ }^{48}$ y que no considera el aporte de otros hemoderivados. El trauma es la causa más frecuente de TM, siendo más frecuente en el trauma bélico, presentándose en $3 \%$ en el trauma civil, aunque este porcentaje aumenta en pacientes que presentan shock hemorrágico hasta $24 \%{ }^{27}$. Independiente de si se trata de trauma bélico o civil, la TM se asocia a una elevada mortalidad, hasta $80 \%$. Además, los pacientes que requieren TM pueden consumir hasta $70 \%$ de las transfusiones en trauma ${ }^{49}$.

El aporte generoso de todos los hemoderivados se basa en la corrección precoz de la CAT. La CAT se asocia a sangrado masivo y riesgo de $\mathrm{TM}^{50}$, por lo que su corrección precoz podría mejorar el pronóstico de los pacientes de trauma grave, evitando fundamentalmente la muerte por sangrado no controlado. Además, modelos farmacocinéticos han establecido que para prevenir la dilución de los factores de coagulación que agrava la CAT, es necesario transfundir el equivalente a sangre total, con una relación plasma fresco congelado (PFC): GR cercana a $1: 1^{51}$. Por otro lado, la evidencia clínica que apoya esta estrategia transfusional tiene limitaciones, siendo mayoritariamente retrospectiva.

Borgman $^{38}$ reportó, en un hospital de campaña, que aquellos pacientes que habían recibido TM y una alta relación plasma: GR $(1: 1,4)$, tenían menor mortalidad que aquellos que habían recibido TM y una relación intermedia $(1: 2,5)$ o baja (1:8). Posteriormente, otros autores confirmaron estos hallazgos en trauma civil. Dushesne ${ }^{40}$, en un estudio retrospectivo en un centro de trauma nivel 1 en Estados Unidos de Norteamérica, encontró una mortalidad de $26 \%$ versus $87,5 \%$ en pacientes con TM que recibieron una relación PFC: GR 1:1 versus 1:4. Holcomb ${ }^{39}$ evaluó el aporte de PFC y plaquetas en 16 centros de trauma nivel 1 en Estados Unidos de Norteamérica, encontrando que aquellos pacientes que habían sido transfundidos con una relación plaquetas: GR mayor a 1:2 y PFC: GR mayor a 1:2, tenían una mejor sobrevida. Stinger ${ }^{41}$ estudió la relación crioprecipitados: GR, reportando que una relación mayor a $0,2 \mathrm{~g}$ de fibrinógeno/1 unidad de GR se asociaba a mejor sobrevida. También se ha estudiado el impacto del uso de protocolos de TM que aseguren una alta relación entre todos los hemoderivados, observando una mejoría en la sobrevida versus controles históricos ${ }^{52-54}$. Parte importante de estos estudios con resultados favorables, han reportado que la mejoría en la sobrevida estaría dada por una disminución de las muertes por sangrado no controlado $38,39,41,52$.

No todos los estudios que han evaluado una alta relación entre hemoderivados han tenido resultados positivos. Kashuk ${ }^{55}$ encontró una disminución en la mortalidad, con una relación PFC: GR entre 1:2 y 1:3, pero con un aumento en ésta con una relación mayor (relación 1:1). Mitra ${ }^{56}$ reportó que una baja relación PFC: GR era un factor de riesgo independiente de mortalidad, perdiéndose este efecto al excluir a los pacientes que murieron durante las primeras $24 \mathrm{~h}$. Este último 
estudio evaluó un punto controversial, ya que el PFC debe ser entibiado antes de administrarse, a diferencia de los GR que pueden administrarse inmediatamente, por lo que la mortalidad precoz podría explicar la baja relación PFC: GR y no viceversa. Por otro lado, varios de los estudios que han reportado una disminución de la mortalidad, también han encontrado un aumento en las complicaciones $^{38,42,43,47}$.

Aún no existe consenso respecto a este manejo transfusional proactivo. En un meta-análisis, Bhangu $^{57}$ encontró que una relación plasma:GR mayor a 1:2 se asoció a una disminución significativa de la mortalidad, sin embargo, una relación 1:1 no tuvo beneficios adicionales. Las "Guías Europeas para el manejo del sangrado después de un trauma mayor" 58 , recomiendan aportar precozmente plasma ( 10 a $15 \mathrm{~mL} / \mathrm{kg})$, sin establecer una relación plasma:GR específica. Lo que sí está claro, es que esta estrategia debe reservarse para los pacientes más graves, que tienen riesgo de sangrado masivo. Como actualmente no existe un examen que permita predecir claramente cuáles pacientes tienen riesgo de sangrado masivo y TM, esta estrategia debe considerarse en los pacientes con riego de $\mathrm{CAT}^{2}$.

Finalmente, los pacientes con traumas menores o que ya se han estabilizado deben manejarse con una estrategia transfusional restrictiva. No se ha demostrado que un aporte liberal de GR en estos pacientes implique una menor mortalidad o morbilidad $^{59}$. Por otro lado, el aporte de plasma a pacientes de trauma sin requerimiento de TM, se ha asociado a mayor morbilidad ${ }^{60}$. Además, deben considerarse los costos de las transfusiones, que podrían llegar a 30\% del total de gastos en UCI en pacientes de trauma ${ }^{61}$.

\section{Conclusiones}

El sangrado no controlado es la causa de muerte prevenible más frecuente del trauma. Para prevenir estas muertes es fundamental corregir precozmente la CAT, mediante la reanimación con control de daños.

No se ha demostrado que ningún fluido sea superior a otro en trauma. Por el momento, los cristaloides isotónicos son los fluidos más seguros y costo-efectivos.

El uso precoz de todos los hemoderivados con una relación entre ellos cercana a 1:1, podría mejorar la sobrevida en los pacientes con sangrado masivo y requerimientos de TM. Estos hallazgos deben corroborarse en ECR, junto con determinar cuál es la relación óptima entre hemoderivados. En espera de estos estudios, parece adecuado aportar en forma precoz y conjunta GR, PFC, plaquetas y crio precipitados, a los pacientes con riesgo de CAT, y por lo tanto, de sangrado masivo y TM. Los pacientes estables deben manejarse con una estrategia transfusional restrictiva.

\section{Referencias}

1. Geeraedts L, Kaasjager H, van Vugt A, Frolke J. Exsanguination in trauma: A review of diagnostics and treatment options. Injury 2009; 40 (1): 11-20.

2. Duchesne J, McSwain N, Cotton B, Hunt J, Dellavolpe J, Lafaro K, et al. Damage Control Resuscitation: The New Face of Damage Control. J Trauma 2010; 69 (4): 976-99.

3. Johansson PI, Stensballe J, Ostrowski SR. Current management of massive hemorrhage in trauma. Scand J Trauma Resusc Emerg Med 2012; 20 (Jul):47. doi: 10.1186/1757-7241-20-47.

4. Dutton RP. Resuscitative strategies to maintain homeostasis during damage control surgery. Br J Surg 2012; 99 (Suppl1): 21-8.

5. Johnson JW, Gracias VH, Schwab CW, Reilly PM, Kauder $\mathrm{DR}$, Shapiro MB, et al. Evolution in damage control for exsanguinating penetrating abdominal injury. J Trauma 2001; 51 (2): 261-9.

6. Brohi K, Singh J, Heron M. Acute traumatic coagulopathy. J Trauma 2003; 54 (6): 1127-30.

7. Maegele M, Lefering R, Yucel N, Tjardes T, Rixen D, Paffrath T, et al. Early coagulopathy in multiple injury: an analysis from the German Trauma Registry on 8724 patients. Injury 2007; 38 (3): 298-304.

8. Floccard B, Rugeri L, Faure A, Saint Denis M, Boyle EM, Peguet $\mathrm{O}$, et al. Early coagulopathy in trauma patients: an on-scene and hospital admission study. Injury 2012; 43 (1): 26-32.

9. Hess J, Brohi K, Dutton R, Hauser C, Holcomb J, Kluger Y, et al. The Coagulopathy of Trauma: A Review of Mechanisms. J Trauma 2008; 65 (4): 748-54.

10. Brohi K, Cohen MJ, Davenport RA. Acute coagulopathy of trauma: mechanism, identification and effect. Curr Opin Crit Care 2007; 13 (6): 680-5.

11. Lier H, Böttiger BW, Hinkelbein J, Krep H, Bernhard M. Coagulation management in multiple trauma: a systematic review. Intensive Care Med 2011; 37 (4): 572-82. 
12. Cushing M, Shaz BH. Blood transfusion in trauma patients: unresolved questions. Minerva Anestesiol 2011; 77 (3): 349-59.

13. Bouillon B, Brohi K, Hess JR, Holcomb JB, Parr MJ, Hoyt DB. Educational initiative on critical bleeding in trauma: Chicago, July 11-13, 2008. J Trauma 2010; 68 (1): 225-30.

14. Sorensen B, Fries D. Emerging treatment strategies for trauma-induced coagulopathy. Br J Surg 2012; 99 (Suppl 1): 40-50.

15. Rugeri L, Levrat A, David JS, Delecroix E, Floccard B, Gros A, et al. Diagnosis of early coagulation abnormalities in trauma patients by rotation thrombelastography. J Thromb Haemost 2007; 5 (2): 289-95.

16. Levrat A, Gros A, Rugeri L, Inaba K, Floccard B, Negrier C, David JS. Evaluation of rotation thrombelastography for the diagnosis of hyperfibrinolysis in trauma patients. Br J Anaesth 2008; 100 (6): 792-7.

17. Sapsford W. Should the 'C' in 'ABCde' Be Altered to reflect the trendtowards hypotensive resuscitation? Scand J Surg 2008; 97 (1): 4-11.

18. Schmidt BM, Rezende-Neto JB, Andrade MV, Winter PC, Carvalho MG Jr, Lisboa TA, et al. Permissive hypotension does not reduce regional organ perfusion compared to normotensive resuscitation: animal study with fluorescent microspheres. World J Emerg Surg 2012; 7 (Suppl 1): S9. doi: 10.1186/1749-7922-7-S1-S9.

19. Li T, Lin X, Zhu Y, Li L, Liu L. Short-term, mild hypothermia can increase the beneficial effect of permissive hypotension on uncontrolled hemorrhagic shock in rats. Anesthesiology 2012; 116 (6): 1288-98.

20. Bickell W, Wall M, Pepe P, Martin R, Ginger V, Allen M, et al. Immediate versus delayed fluid resuscitation for hypotensive patients with penetrating torso injuries. $\mathrm{N}$ Engl J Med 1994; 331 (17): 1105-9.

21. Dutton R, Mackenzie C, MD, Scalea T. Hypotensive Resuscitation during Active Hemorrhage: Impact on In-Hospital Mortality. J Trauma 2002; 52 (6): 1141-6.

22. Morrison C, Carrick M, Norman M, Scott B, Welsh F, Tsai P, et al. Resuscitation Strategy Reduces Transfusion Requirements and Severe Postoperative Coagulopathy in Trauma Patients With Hemorrhagic Shock: Preliminary Results of a Randomized Controlled Trial. J Trauma 2011; 70 (3): 652-63.

23. Chiara O, Bucci L, Sara A, Bassi G, Vesconi S. Quality and quantity of volumen replacement in trauma patients. Minerva Anestesiol 2008; 74 (6): 303-6.

24. Ley E, Clond M, Srour M, Barnajian M, Mirocha J, MS, Margulies D, et al. Emergency Department Crystalloid Resuscitation of 1.5 L or More is Associated With Increased Mortality in Elderly and Nonelderly Trauma Patients. J Trauma 2011; 70 (2): 398-400.
25. Haut E, Kalish B, Cotton B, Efron D, Haider A, Stevens $\mathrm{K}$, et al. Prehospital Intravenous Fluid Administration Is Associated With Higher Mortality in Trauma Patients: A National Trauma Data Bank Analysis. Ann Surg 2011; 253 (2): 371-8.

26. Bouglé A, Harrois A, Duranteau J. Resuscitative strategies in traumatic hemorrhagicshock. Ann Intensive Care 2013; 3 (1): 1. doi: 10.1186/2110-5820-3-1.

27. Gruen R, Brohi K, Schreiber M, Balogh Z, Pitt V, Narayan $\mathrm{M}$, et al. Haemorrhage control in severely injured patients. Lancet 2012; 380 (9847): 1099-108.

28. Homma H, Deitch E, Feketeova E, Lu Q, Berezina T, Zaets S, et al. Small Volume Resuscitation With Hypertonic Saline Is More Effective in Ameliorating Trauma-Hemorrhagic Shock-Induced Lung Injury, Neutrophil Activation and Red Blood Cell Dysfunction than Pancreatic Protease Inhibition. J Trauma 2005; 59 (2): 266-72.

29. Rizoli S, Rhind S, Shek P, Inaba K, Filips D, Tien H. The Immunomodulatory Effects of Hypertonic Saline Resuscitation in Patients Sustaining Traumatic Hemorrhagic Shock. A Randomized, Controlled, Double-Blinded Trial. Ann Surg 2006; 243 (1): 47-57.

30. Junger WG, Rhind SG, Rizoli SB, Cuschieri J, Shiu MY, Baker AJ, et al. Resuscitation of traumatic hemorrhagic shock patients with hypertonic saline-without dextraninhibits neutrophil and endothelial cell activation. Shock 2012; 38 (4): 341-50.

31. Bulger E, Jurkovich J, Nathens A, Copass M, Hanson S, Cooper C, et al. Hypertonic Resuscitation of Hypovolemic Shock After Blunt Trauma. A Randomized Controlled Trial. Arch Surg 2008; 143 (2): 139-48.

32. Bulger E, May S, Kerby J, Emerson S, Stiell I, Schreiber $\mathrm{M}$, et al. Out-of-hospital Hypertonic Resuscitation After Traumatic Hypovolemic Shock: A Randomized, Placebo Controlled Trial. Ann Surg 2011; 253 (3): 431-41.

33. Perner A, Haase N, Guttormsen AB, Tenhunen J, Klemenzson G, Åneman A, et al. Hydroxyethyl starch $130 / 0.42$ versus Ringer's acetate in severe sepsis. N Engl J Med 2012; 367 (2): 124-34

34. Myburgh J, Finfer S, Bellomo R, Billot L, Cass A, Gattas D, et al. Hydroxyethyl Starch or Saline for Fluid Resuscitation in Intensive Care. N Engl J Med 2012; 367 (20): 1901-11.

35. Reinhart K, Perner A, Sprung CL, Jaeschke R, Schortgen F, Johan Groeneveld $\mathrm{AB}$, et al. Consensus statement of the ESICM task force on colloid volume therapy in critically ill patients. Intensive Care Med 2012; 38 (3): 368-83.

36. Perel P, Roberts I, Ker K. Colloids versus crystalloids for fluid resuscitation in critically ill patients. Cochra- 
ne Database of Systematic Reviews 2013, Issue 2. Art. No.: CD000567. DOI: 10.1002/14651858.CD000567. pub6.

37. Finfer S, Bellomo R, Boyce N, French J, Myburgh J, Norton R, et al. Comparison of Albumin and Saline for Fluid Resuscitation in the Intensive Care Unit. N Engl J Med 2004; 350 (22): 2247-56.

38. Borgman MA, Spinella PC, Perkins JG, Grathwohl KW, Repine T, Beekley AC, et al. The ratio of blood products transfused affects mortality in patients receiving massive transfusions at a combat support hospital. J Trauma 2007; 63 (4): 805-13.

39. Holcomb JB, Wade CE, Michalek JE, Chisholm GB, Zarzabal LA, Schreiber MA, et al. Increased plasma and platelet to red blood cell ratios improves outcome in 466 massively transfused civilian trauma patients. Ann Surg 2008; 248 (3): 447-58.

40. Duchesne JC, Hunt JP, Wahl G, Marr AB, Wang YZ, Weintraub SE, et al. Review of current blood transfusions strategies in a mature level I trauma center: were we wrong for the last 60 years? J Trauma 2008; 65 (2): 272-6.

41. Stinger HK, Spinella PC, Perkins JG, Grathwohl KW, Salinas J, Martini WZ, et al. The ratio of fibrinogen to red cells transfused affects survival in casualties receiving massive transfusions at an army combat support hospital. J Trauma 2008; 64 (2 Suppl): S79-85.

42. Maegele M, Lefering R, Paffrath T, Tjardes T, Simanski C, Bouillon B, et al. Red-blood-cell to plasma ratios transfused during massive transfusion are associated with mortality in severe multiple injury: a retrospective analysis from the Trauma Registry of the Deutsche Gesellschaft für Unfallchirurgie. Vox Sang 2008; 95 (2): 112-9.

43. Sperry JL, Ochoa JB, Gunn SR, Alarcón LH, Minei JP, Cuschieri J, et al. An FFP: PRBC transfusion ratio $>/=1: 1.5$ is associated with a lower risk of mortality after massive transfusion. J Trauma 2008; 65 (5): 986-93.

44. Perkins JG, Cap AP, Spinella PC, Blackbourne LH, Grathwohl KW, Repine TB, et al. An evaluation of the impact of apheresis platelets used in the setting of massively transfused trauma patients. J Trauma 2009; 66 (4 Suppl): S77-84.

45. Zink KA, Sambasivan CN, Holcomb JB, Chisholm G, Schreiber MA. A high ratio of plasma and platelets to packed red blood cells in the first 6 hours of massive transfusion improves outcomes in a large multicenter study. Am J Surg 2009; 197 (5): 565-70.

46. Shaz BH, Dente CJ, Nicholas J, MacLeod JB, Young AN, Easley $\mathrm{K}$, et al. Increased number of coagulation products in relationship to red blood cell products transfu- sed improves mortality in trauma patients. Transfusion 2010; 50 (2): 493-500.

47. Holcomb JB, Zarzabal LA, Michalek JE, Kozar RA, Spinella PC, Perkins JG, et al. Increased platelet: RBC ratios are associated with improved survival after massive transfusion. J Trauma 2011; 71 (2 Suppl 3): S318-28.

48. Reed MJ, Lone N, Walsh TS. Resuscitation of the trauma patient: tell me a trigger for early haemostatic resuscitation please! Crit Care 2011; 15 (2): 126. doi: 10.1186/ cc10014.

49. Sihler KC, Napolitano LM. Massive transfusion: new insights. Chest 2009; 136 (6): 1654-67.

50. Frith D, Brohi K. The pathophysiology of traumainduced coagulopathy. Curr Opin Crit Care 2012; 18 (6): 631-6.

51. Ho AM, Dion PW, Cheng CA, Karmakar MK, Cheng G, Peng Z, et al. A mathematical model for fresh frozen plasma transfusion strategies during major trauma resuscitation with ongoing hemorrhage. Can J Surg 2005; 48 (6): 470-8.

52. Dente CJ, Shaz BH, Nicholas JM, Harris RS, Wyrzykowski $\mathrm{AD}$, Patel $\mathrm{S}$, et al. Improvements in early mortality and coagulopathy are sustained better in patients with blunt trauma after institution of a massive transfusion protocol in a civilian level I trauma center. J Trauma 2009; 66 (6): 1616-24.

53. Johansson PI, Stensballe J. Effect of Haemostatic Control Resuscitation on mortality in massively bleeding patients: a before and after study. Vox Sang 2009; 96 (2): $111-8$.

54. Gunter OL Jr, Au BK, Isbell JM, Mowery NT, Young $\mathrm{PP}$, Cotton BA. Optimizing outcomes in damage control resuscitation: identifying blood product ratios associated with improved survival. J Trauma 2008; 65 (3): 527-34.

55. Kashuk JL, Moore EE, Johnson JL, Haenel J, Wilson M, Moore JB, et al. Post injury life threatening coagulopathy: is 1:1 fresh frozen plasma:packed red blood cells the answer? J Trauma 2008; 65 (2): 261-70.

56. Mitra B, Mori A, Cameron PA, Fitzgerald M, Paul E, Street A. Fresh frozen plasma (FFP) use during massive blood transfusion in trauma resuscitation. Injury 2010; 41 (1): 35-9.

57. Bhangu A, Nepogodiev D, Doughty H, Bowley D. Metaanalysis of plasma to red blood cell ratios and mortality in massive blood transfusions for trauma. Injury 2012; 7 (Sept): 193-9.

58. Rossaint R, Bouillon B, Cerny V, Coats TJ, Duranteau J, Fernández-Mondéjar, et al. Management of bleeding following major trauma: an updated European guideline. Crit Care 2010; 14 (2): R52. doi: 10.1186/cc8943. 
59. McIntyre L, Hebert PC, Wells G, Fergusson D, Marshall J, Yetisir E, et al. Is a restrictive transfusion strategy safe for resuscitated and critically ill trauma patients? J Trauma 2004; 57 (3): 563-8.

60. Inaba K, Branco BC, Rhee P, Blackbourne LH, Holcomb JB, Teixeira PG, et al. Impact of plasma transfusion in trauma patients who do not require massive transfusion. J Am Coll Surg 2010; 210 (6): 957-65.

61. Pape HC, Neugebauer E, Ridley SA, Chiara O, Nielsen TG, Christensen MC. Cost-drivers in acute treatment of severe trauma in Europe: a systematic review of literature. Eur J Trauma Emerg Surg 2009; 35 (1) 61-6. 